\title{
The spectrum of low spin mesons at finite temperature in holographic noncommutative QCD
}

\author{
Tadahito NAKAJIMA, ${ }^{*}$ Yukiko OHTAKE ${ }^{\dagger}$ and Kenji SUZUKI ${ }^{\ddagger}$ \\ * College of Engineering, Nihon University, Fukushima 963-8642, Japan \\ † Toyama National College of Technology, Toyama 939-8630, Japan \\ $\ddagger$ Department of Physics, Ochanomizu University, Tokyo 112-8610, Japan
}

\begin{abstract}
We have constructed a noncommutative deformation of the holographic QCD (Sakai-Sugimoto) model and evaluated the mass spectrum of low spin vector mesons at finite temperature. The masses of light vector- and pseudovector-meson in the noncommutative holographic QCD model reduces over the whole area in the intermediate-temperature regime compared to the commutative case. However, the space noncommutativity does not change the properties of temperature dependence for the mass spectrum of low spin mesons. The masses of meson also decrease with increasing temperature in noncommutative case.
\end{abstract}




\section{Introduction}

Noncommutative gauge theories (gauge theories on noncommutative space) naturally arise as low energy theories of D-branes in Neveu-Schwarz-Neveu-Schwarz (NS-NS) B-field background [1, 2, 3, 4, 5]. The space noncommutativity brings nontrivial properties on the gauge field theory at the quantum level. A remarkable example is the mixing between the infrared and the ultraviolet degrees of freedom. Although the product of the momentum and the noncommutativity parameter plays the role of ultraviolet cut-off, the result is singular when the momentum or the noncommutativity parameter is taken to zero. This phenomenon is referred to as the UV/IR mixing [6]. Although the noncommutative gauge theories have been studied extensively, it is hard to investigate them in the perturbative approach. Little is currently known of the non-perturbative properties of noncommutative gauge theories.

The noncommutative gauge theories have gravity duals whose near horizon region describes the large $N$ limit of the noncommutative gauge theories [7, 8, 9]. Based on the noncommutative deformation of gauge/gravity duality, we can investigate the non-perturbative aspects of the large $N$ limit of the noncommutative gauge theories. For instance, the space noncommutativity modifies the Wilson loop behavior [10, 11, 12] and glueball mass spectra [13]. The gravity duals of noncommutative gauge theories with matter in the fundamental representation have also been constructed by adding probe flavor branes [14]. Employing the gravity dual description of noncommutative gauge theories with flavor degrees of freedom we have been able to find the space noncommutativity is also reflected in the flavor dynamics. For instance, the mass spectrum of mesons can be modified by the space noncommutativity [14].

Fundamental properties of quantum chromodynamics (QCD) at low energies are confinement and chiral symmetry breaking. The holographic QCD model (D4-D8- $\overline{\mathrm{D} 8}$ model) so-called Sakai-Sugimoto model has been known to capture these properties of QCD at low energies [15, 16]. The holographic QCD models can be modified to introduce a finite temperature. The phase transition of chiral symmetry restoration could be described in terms of the holographic QCD at finite temperature. The holographic QCD at finite temperature has three phase structures, confined with broken chiral symmetry, deconfined with restored chiral symmetry, and deconfined with broken chiral symmetry [17, 18]. The finite baryon chemical potential and density can be also naturally introduced in the holographic QCD at finite temperature and bring the rich structure in this model [19, 20, 21, 22, 23]. The properties of mesons at finite temperature has been analyzed within the framework of the holographic QCD at finite temperature [24, 25, 26]. How does the influence of the space noncommutativity reflect the properties of the mesons in the QCD at finite temperature.

In this paper, we construct the noncommutative deformation of the holographic QCD at finite temperature in imitation of the method by Alishahiha et al. [9] and Arean et al. [14] and investigate the influence of space noncommutativity on the mass spectrum of low spin mesons 
in the QCD at finite temperature. There are some attempts to investigate the nontrivial effect of the space noncommutativity on the QCD in the context of the noncommutative deformation of the holographic QCD [29, 30]. As will be seen later, the space noncommutativity is also able to modify the mass spectrum of meson.

This paper is organized as follows. In section 2, we construct the noncommutative deformation of the holographic QCD model at finite temperature. In section 3, we analyze the mass spectrum of low-spin meson in the intermediate-temperature phase, which is the phase of deconfined with broken chiral symmetry within the framework of the noncommutative deformation of the holographic QCD model. Section 4 is devoted to conclusions.

\section{Noncommutative deformation of the holographic QCD model at finite temperature}

In this section, we consider a noncommutative deformation of the holographic QCD (SakaiSugimoto) model at finite temperature based on the prescription of Arean-Paredes-Ramallo [14]. The holographic QCD model is a gravity dual for a $4+1$ dimensional QCD with $\left.\mathrm{U}_{(} \mathrm{N}_{f}\right)_{L} \times$ $\mathrm{U}\left(\mathrm{N}_{f}\right)_{R}$ global chiral symmetry whose symmetry is spontaneously broken [15, 16]. This model is a D4-D8- $\overline{\mathrm{D} 8}$-brane system consisting $S^{1}$ compactified $N_{c}$ D4-branes and $N_{f}$ D8- $\overline{\mathrm{D} 8}$ pairs transverse to the $S^{1}$. The near-horizon limit of the set of $N_{c}$ D4-branes solution compactified on $S^{1}$ takes the form

$$
\begin{aligned}
d s^{2} & =\left(\frac{U}{R_{\mathrm{D} 4}}\right)^{3 / 2}\left(-(d t)^{2}+\left(d x^{1}\right)^{2}+\left(d x^{2}\right)^{2}+\left(d x^{3}\right)^{2}+f_{\mathrm{KK}}(U) d \tau^{2}\right) \\
& +\left(\frac{R_{\mathrm{D} 4}}{U}\right)^{3 / 2}\left(\frac{d U^{2}}{f_{\mathrm{KK}}(U)}+U^{2} d \Omega_{4}^{2}\right) \\
& R_{\mathrm{D} 4}^{3}=\pi g_{s} N_{c} l_{s}^{3}, \quad f_{\mathrm{KK}}(U)=1-\frac{U_{\mathrm{KK}}{ }^{3}}{U^{3}}
\end{aligned}
$$

where $U_{\mathrm{KK}}$ is a parameter, $U$ is the radial direction bounded from below by $U \geq U_{\mathrm{KK}}, \tau$ is compactified direction of the D4-brane world volume which is transverse to the D8-D8-branes, $g_{s}$ and $l_{s}$ are the string coupling and length respectively. The dilaton $\phi$ and the field strength $F_{4}$ of the RR 3-form $C_{3}$ are given by

$$
e^{\phi}=g_{s}\left(\frac{U}{R_{\mathrm{D} 4}}\right)^{3 / 4}, \quad F_{4}=d C_{3}=\frac{2 \pi N_{c}}{V_{4}} \epsilon_{4},
$$

where $V_{4}=8 \pi^{2} / 3$ is the volume of unit $S^{4}$ and $\epsilon_{4}$ is the corresponding volume form. In order to avoid a conical singularity at $U=U_{\mathrm{KK}}$, the $\tau$ direction should have a period of

$$
\delta \tau=\frac{4 \pi}{3}\left(\frac{R_{\mathrm{D} 4}^{3}}{U_{\mathrm{KK}}}\right)^{1 / 2}=2 \pi R=\frac{2 \pi}{M_{\mathrm{KK}}},
$$


where $R$ is radius of $S^{1}$ and $M_{\mathrm{KK}}$ is the Kaluza-Klein mass. The parameter $U_{\mathrm{KK}}$ is related to the Kaluza-Klein mass $M_{\mathrm{KK}}$ via the relation (2.3). The five dimensional gauge coupling is expressed in terms of $g_{s}$ and $l_{s}$ as $g_{\mathrm{YM}}^{2}=(2 \pi)^{2} g_{s} l_{s}$. The gravity description is valid for strong coupling $\lambda \gg R$, where as usual $\lambda=g_{\mathrm{YM}}^{2} N_{c}$ denotes the 't Hooft coupling.

The holographic QCD model at finite temperature has been proposed in [17, 18, 24]. In order to introduce a finite temperature $T$ in the model, we consider the Euclidean gravitational solution which is asymptotically equals to (2.1) but with the compactification of Euclidean time direction $t_{E}$. In this solution the periodicity of $t_{E}$ is arbitrary and equals to $\beta=1 / T$.

Another solution with the same asymptotic is given by interchanging the role of $t_{E}$ and $\tau$ directions,

$$
\begin{aligned}
d s^{2} & =\left(\frac{U}{R_{\mathrm{D} 4}}\right)^{3 / 2}\left(f_{\mathrm{T}}(U)\left(d t_{E}\right)^{2}+\left(d x^{1}\right)^{2}+\left(d x^{2}\right)^{2}+\left(d x^{3}\right)^{2}+d \tau^{2}\right) \\
& +\left(\frac{R_{\mathrm{D} 4}}{U}\right)^{3 / 2}\left(\frac{d U^{2}}{f_{\mathrm{T}}(U)}+U^{2} d \Omega_{4}^{2}\right) \\
& R_{\mathrm{D} 4}^{3}=\pi g_{s} N_{c} l_{s}^{3}, \quad f_{\mathrm{T}}(U)=1-\frac{U_{T}^{3}}{U^{3}},
\end{aligned}
$$

where $U_{T}$ is a parameter. To avoid a singularity at $U=U_{T}$ the period of $\delta t_{E}$ of the compactified time direction is set to

$$
\delta t_{E}=\frac{4 \pi}{3}\left(\frac{R_{\mathrm{D} 4}^{3}}{U_{T}}\right)^{1 / 2}=\frac{1}{T},
$$

and the parameter $U_{T}$ is related to the temperature $T$. The metric (2.1) with the compactification of Euclidean time $t_{E}$ is dominant in the low temperature $T<1 / 2 \pi R$, while the metric (2.4) is dominant in the high temperature $T>1 / 2 \pi R$. The transition between the metric (2.1) and the metric (2.4) happens when $T=T_{c}=1 / 2 \pi R$. This transition is first-order and corresponds to the confinement/deconfinement phase transition in the gauge theory side.

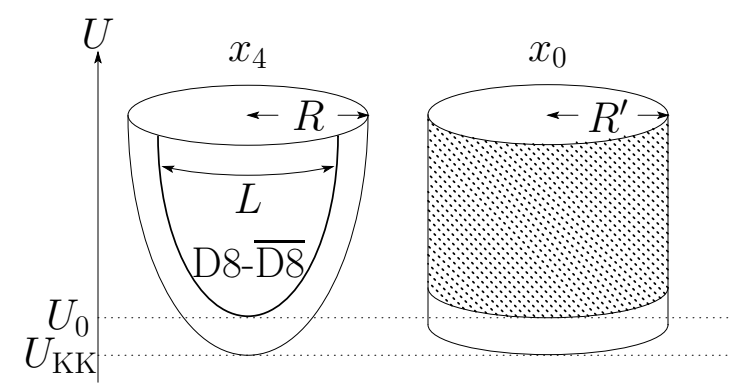

Fig. 1 The D8-D8-branes configurations at low temperature. 

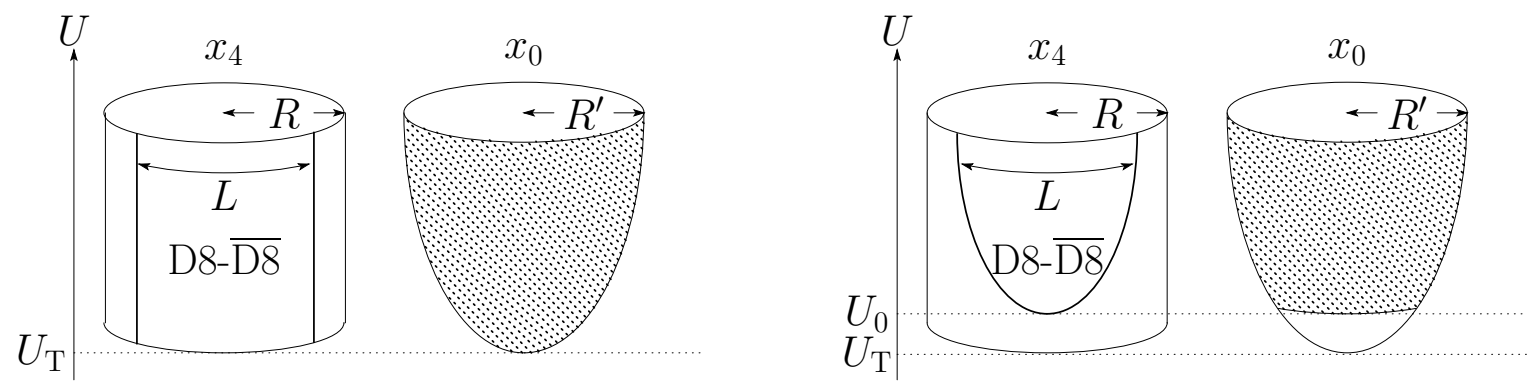

Fig. 2 The D8- $\overline{\mathrm{D} 8}$-branes configurations at high temperature.

In the low temperature, the D8- and $\overline{\mathrm{D} 8}$-branes are connected at $U=U_{0}$ as shown in Fig. 1. The connected configuration of the D8-D $\overline{\mathrm{D}} 8$-branes indicates that the $\mathrm{U}\left(\mathrm{N}_{f}\right)_{L} \times \mathrm{U}\left(\mathrm{N}_{f}\right)_{R}$ global chiral symmetry is broken to a diagonal subgroup $\mathrm{U}\left(\mathrm{N}_{f}\right)$. We refer to the connected configuration in the low temperature as the low-temperature phase. In the high temperature, there are two kinds of configurations as shown in Fig. 2. One is connected configuration and the other is disconnected configuration that the D8- and $\overline{\mathrm{D} 8}$-branes hang vertically from infinity down to the horizon. The disconnected configuration of the D8- $\overline{\mathrm{D}} 8$-branes indicates that the $\mathrm{U}\left(\mathrm{N}_{f}\right)_{L} \times \mathrm{U}\left(\mathrm{N}_{f}\right)_{R}$ global chiral symmetry is restored. We refer to the disconnected configuration and the connected configuration in the high temperature as the high-temperature phase and the intermediate-temperature phase, respectively. The intermediate-temperature phase is realized when the confinement/deconfinement phase transition and the chiral phase transition does not occur simultaneously.

When turning on a NS-NS $B$-field on the D-brane worldvolume, the low-energy effective worldvolume theories are deformed to a noncommutative Yang-Mills theories [1, 2, 3, 4, 5]. The D-brane realizations of noncommutative Yang-Mills theories have a gravity dual in the large $N$, strong 't Hooft coupling limit [7, 8, 9]. In accordance with the formulation of [7, 8, 9], we attempt to construct the gravity dual of the noncommutative QCD whose chiral symmetry is spontaneously broken by deforming the holographic QCD model. Let us consider the D4branes solution compactified on a circle in the $\tau$-direction. T-dualizing it along $x^{3}$ produces a D3-branes delocalized along $x^{3}$. After rotating the D3-branes along the $\left(x^{2}, x^{3}\right)$ plane, we T-dualize back on $x^{3}$. This procedure yields the solution with a $B_{23}$ fields along the $x^{2}$ and $x^{3}$ directions. The solution in the low temperature takes the form

$$
\begin{aligned}
d s^{2} & =\left(\frac{U}{R_{\mathrm{D} 4}}\right)^{3 / 2}\left(\left(d t_{E}\right)^{2}+\left(d x^{1}\right)^{2}+h\left\{\left(d x^{2}\right)^{2}+\left(d x^{3}\right)^{2}\right\}+f_{\mathrm{KK}}(U) d \tau^{2}\right) \\
& +\left(\frac{R_{\mathrm{D} 4}}{U}\right)^{3 / 2}\left(\frac{d U^{2}}{f_{\mathrm{KK}}(U)}+U^{2} d \Omega_{4}^{2}\right),
\end{aligned}
$$

where $h(U)=\frac{1}{1+\theta^{3} U^{3}}$ and $\theta$ denotes the noncommutativity parameter with dimension of [length $]^{-1}$. When $\theta \neq 0$ this solution is dual to a gauge theory in which the coordinates $x^{2}$ and 
$x^{3}$ do not commute. It is obvious that this solution reduces to the solution (2.1) with Euclidean signature when $\theta=0$. In the high temperature, the solution (2.6) changes to

$$
\begin{aligned}
d s^{2} & =\left(\frac{U}{R_{\mathrm{D} 4}}\right)^{3 / 2}\left(f_{\mathrm{T}}(U)\left(d x_{E}\right)^{2}+\left(d x^{1}\right)^{2}+h\left\{\left(d x^{2}\right)^{2}+\left(d x^{3}\right)^{2}\right\}+d \tau^{2}\right) \\
& +\left(\frac{R_{\mathrm{D} 4}}{U}\right)^{3 / 2}\left(\frac{d U^{2}}{f_{\mathrm{T}}(U)}+U^{2} d \Omega_{4}^{2}\right) .
\end{aligned}
$$

The solution has the same form as the one in the low temperature (2.6), but with the role of the $\tau$ and $t_{E}$ directions exchanged.

\section{Mass spectrum of low spin mesons in the intermediate- temperature phase}

In order to analyze the phases of QCD at finite temperature, we needs to determine the shape of the D8-brane whose effective action is given by the DBI action and the WZ action:

$$
\begin{aligned}
& S^{\mathrm{D} 8}=S_{\mathrm{DBI}}^{\mathrm{D} 8}+S_{\mathrm{WZ}}^{\mathrm{D} 8}, \\
& S_{\mathrm{DBI}}^{\mathrm{D} 8}=T_{8} \int d^{9} x e^{-\phi} \operatorname{Tr} \sqrt{\operatorname{det}\left(g_{M N}+B_{M N}+2 \pi \alpha^{\prime} F_{M N}\right)}, \\
& S_{\mathrm{WZ}}^{\mathrm{D} 8}=\mu_{8} \int_{\mathrm{D} 8} C_{3} \wedge e^{\left(\widetilde{B}+2 \pi l_{s}^{2} F\right)},
\end{aligned}
$$

where $T_{8}$ is the tension of the D8-brane, $\mu_{8}$ is the D8-brane charge, $\phi$ is a dilaton and $\widetilde{B}=$ $B_{M N} d x^{M} d x^{N}$ is a NS-NS $B$-field:

$$
\begin{aligned}
& e^{2 \phi}=g_{s}{ }^{2} h(U)\left(\frac{U}{R_{\mathrm{D} 4}}\right)^{3 / 4}, \\
& B_{M N}(U)= \begin{cases}\theta^{3 / 2} \frac{U^{3}}{R_{\mathrm{D} 4}^{3 / 2}} h(U) & (M=2, N=3) \\
0 & \text { (others) }\end{cases}
\end{aligned}
$$

We examine the spectrum of low-spin mesons in the intermediate-temperature phase. Let us consider the background metric (2.7). This leads to an induced metric on D8-brane worldvolume,

$$
\begin{aligned}
d \bar{s}_{\mathrm{I}}^{2} & =\left(\frac{U}{R_{\mathrm{D} 4}}\right)^{3 / 2}\left(f_{\mathrm{T}}(U)\left(d x_{E}\right)^{2}+\left(d x^{1}\right)^{2}+h\left\{\left(d x^{2}\right)^{2}+\left(d x^{3}\right)^{2}\right\}\right) \\
& +\left[\left(\frac{U}{R_{\mathrm{D} 4}}\right)^{3 / 2}\left(\tau^{\prime}(U)\right)^{2}+\left(\frac{R_{\mathrm{D} 4}}{U}\right)^{3 / 2} \frac{1}{f_{\mathrm{T}}(U)}\right] d U^{2}+\left(\frac{R_{\mathrm{D} 4}}{U}\right)^{3 / 2} U^{2} d \Omega_{4}^{2}
\end{aligned}
$$


where $\tau^{\prime}(U)$ denotes the derivative of $\tau(U)$ with respect to $U$. Expanding the (abelian) DBI action with respect to $F_{M N}$, we have

$$
S_{\mathrm{D} 8}^{\mathrm{DBI}}=T_{8} \int d^{9} x e^{-\phi} \sqrt{\operatorname{det}\left(g_{M N}+B_{M N}\right)}\left\{1+\frac{1}{4}\left(2 \pi \alpha^{\prime}\right)^{2} g^{J K} g^{P Q} F_{J P} F_{K Q}+\mathcal{O}\left(F^{4}\right)\right\} .
$$

We set $A_{\alpha}(\alpha=5,6,7,8,9)=0$ and assume that $A_{\mu}(\mu=0,1,2,3)$ and $A_{U}$ are independent of the coordinates on the $S^{4}$.

Under the induced metric (3.4) and the dilaton (3.2), the DBI action for the zeroth order in $\alpha^{\prime}$

$$
S_{\mathrm{DBI}}^{\mathrm{D} 8(0)}=T_{8} \int d^{9} x e^{-\phi} \sqrt{\operatorname{det}\left(g_{M N}+B_{M N}\right)}
$$

takes the form

$$
S_{\mathrm{DBI}}^{\mathrm{D} 8(0)}=\frac{N_{f} T_{8} V_{4}}{g_{s}} \int d^{4} x d U U^{4} \sqrt{f_{\mathrm{T}}(U)\left(\tau^{\prime}(U)\right)^{2}+\left(\frac{R_{\mathrm{D} 4}}{U}\right)^{3}}
$$

where $N_{f}$ is the number of the flavor and $V_{4}=\frac{8 \pi^{2}}{3}$ denotes the volume form of the unit 4sphere. The action (3.7) does not depend on the noncommutativity parameter $\theta$, though the action (3.6) includes the $B$-field (3.3). The equation of motion for $\tau$ is

$$
\frac{d}{d U}\left[\frac{U^{4} f_{\mathrm{T}}(U)\left(\tau^{\prime}(U)\right)}{\sqrt{f_{\mathrm{T}}(U)\left(\tau^{\prime}(U)\right)^{2}+\left(\frac{R_{\mathrm{D} 4}}{U}\right)^{3}}}\right]=0 .
$$

We impose the condition $\lim _{U \rightarrow U_{0}} \tau^{\prime}(U) \rightarrow \infty$ that corresponds to the connected configuration of the D8- $\overline{\mathrm{D} 8}$-branes.

The solution of (3.8) under the condition $\lim _{U \rightarrow U_{0}} \tau^{\prime}(U) \rightarrow \infty$ is given by

$$
\tau(U)=U_{0}^{4} \sqrt{f_{\mathrm{K}}\left(U_{0}\right)} \int_{U_{0}}^{U} d U \frac{\left(\frac{R_{\mathrm{D} 4}}{U}\right)^{3 / 2}}{\sqrt{f_{\mathrm{K}}(U)} \sqrt{U^{8} f_{\mathrm{K}}(U)-U_{0}^{8} f_{\mathrm{K}}\left(U_{0}\right)}} .
$$

Using the solution (3.9), we have

$$
\left(\frac{U}{R_{\mathrm{D} 4}}\right)^{3 / 2}\left(\tau^{\prime}(U)\right)^{2}+\left(\frac{R_{\mathrm{D} 4}}{U}\right)^{3 / 2} \frac{1}{f_{\mathrm{T}}(U)}=\left(\frac{R_{\mathrm{D} 4}}{U}\right)^{3 / 2} \frac{U^{8}}{U^{8} f_{\mathrm{T}}(U)-U_{0}^{8} f_{\mathrm{T}}\left(U_{0}\right)} .
$$

The DBI action (3.5) with second-order in $\alpha^{\prime}$ can be rewritten by substituting (3.7) as

$$
\begin{aligned}
S_{\mathrm{DBI}}^{\mathrm{D} 8(2)} & =\frac{N_{f} T_{8} V_{4}}{g_{s}} \frac{\left(2 \pi l_{s}^{2}\right)^{2}}{2} \int d^{4} x d U f_{\mathrm{T}}(U)^{1 / 2} U^{4}\left(\frac{R_{\mathrm{D} 4}}{U}\right)^{3 / 2} k(U)^{1 / 2} \\
& \times\left[\left(\frac{R_{\mathrm{D} 4}}{U}\right)^{3} f_{\mathrm{T}}(U)^{-1}\left\{F_{01}^{2}+\frac{1}{h(U)}\left(F_{02}^{2}+F_{03}^{2}\right)\right\}\right. \\
& \left.+\frac{1}{k(U) f_{\mathrm{T}}(U)} F_{02}^{2}+\frac{1}{k(U)^{2}} F_{1 U}^{2}+\frac{1}{k(U) h(U)}\left(F_{2 U}^{2}+F_{3 U}^{2}\right)\right] .
\end{aligned}
$$


where $k(U)=\frac{U^{8}}{U^{8} f_{\mathrm{T}}(U)-U_{0}^{8} f_{\mathrm{T}}(U)}$. The WZ action is rewritten as

$$
S_{\mathrm{WZ}}^{\mathrm{D} 8(2)}=\frac{1}{3} \frac{N_{c} N_{f}}{(2 \pi)^{3} l_{s}^{2}} \int d^{4} x d U B_{23}\left\{A_{0} F_{1 U}-A_{1} F_{0 U}+A_{U} F_{01}\right\} .
$$

The total action is given as follows

$$
\begin{aligned}
& S^{\mathrm{D} 8(2)}=S_{\mathrm{DBI}}^{\mathrm{D} 8(2)}+S_{\mathrm{WZ}}^{\mathrm{D} 8(2)} \\
& =\frac{N_{f} T_{8} V_{4}}{g_{s}} \frac{\left(2 \pi l_{s}^{2}\right)^{2}}{2} \int d^{4} x d U\left[\left(\frac{R_{\mathrm{D} 4}}{U}\right)^{3 / 2} k(U)^{1 / 2} f_{\mathrm{T}}(U)^{-1 / 2}\left\{F_{01}^{2}+\frac{1}{h(U)}\left(F_{02}^{2}+F_{03}^{2}\right)\right\}\right. \\
& +\left(\frac{U}{R_{\mathrm{D} 4}}\right)^{3} k(U)^{-1 / 2}\left\{f_{\mathrm{T}}(U)^{-1 / 2} F_{0 U}^{2}+f_{\mathrm{T}}(U)^{1 / 2} F_{1 U}^{2}+\frac{f_{\mathrm{T}}(U)^{1 / 2}}{h(U)}\left(F_{2 U}^{2}+F_{3 U}^{2}\right)\right\} \\
& \left.+\kappa\left(\frac{U}{R_{\mathrm{D} 4}}\right)^{3 / 2} \frac{N(\Theta, U)}{\left(U / U_{0}\right)^{5 / 2}}\left\{A_{0} F_{1 U}-A_{1} F_{0 U}+A_{U} F_{01}\right\}\right],
\end{aligned}
$$

where $\kappa=2 / U_{0}, N(\Theta, U)=\frac{\Theta^{3 / 2}\left(U / U_{0}\right)^{3}}{1+\Theta^{3}\left(U / U_{0}\right)^{3}}$ and $\Theta=U_{0} \theta$ denotes the dimensionless noncommutativity parameter. In deriving the coefficient of $(\underline{3.13})$, we utilize $T_{8}=\frac{1}{(2 \pi)^{8} l_{s}^{9}}$ and $R_{\mathrm{D} 4}^{3}=\pi d_{s} N_{c} l_{s}^{3}$.

To simplify the consideration, we choose to focus on the components $A_{1}$ and $A_{U}$, and suppose that these gauge fields do not depend on the spatial coordinates $x_{1}, x_{2}$ and $x_{3}$. The equations of motion for $A_{1}$ and $A_{U}$ are given by

$$
\begin{aligned}
& \partial_{U}\left\{2 k(U)^{-1 / 2} f_{\mathrm{T}}(U)^{1 / 2}\left(\frac{U}{R_{\mathrm{D} 4}}\right)^{3 / 2} \partial_{U} A_{1}\right\}-2 k(U)^{1 / 2} f_{\mathrm{T}}(U)^{-1 / 2}\left(\frac{R_{\mathrm{D} 4}}{U}\right)^{3 / 2} \partial_{0}^{2} A_{1} \\
& +2 \kappa\left(\frac{U}{R_{\mathrm{D} 4}}\right)^{3 / 2} \frac{N(\Theta, U)}{\left(U / U_{0}\right)^{5 / 2}} F_{0 U}-\partial_{U}\left\{\kappa\left(\frac{U}{R_{\mathrm{D} 4}}\right)^{3 / 2} \frac{N(\Theta, U)}{\left(U / U_{0}\right)^{5 / 2}}\right\} A_{0}=0 \\
& f_{\mathrm{T}}(U)^{-1 / 2} \partial_{0} F_{0 U}-\kappa k(U)^{1 / 2}\left(U / U_{0}\right)^{-5 / 2} \frac{N(\Theta, U)}{\left(U / U_{0}\right)^{5 / 2}} \partial_{0} A_{1}=0 .
\end{aligned}
$$

Eliminating $F_{0 U}$ from (3.14) and (3.15), we have

$$
\begin{aligned}
& \partial_{U}\left\{2 k(U)^{-1 / 2} f_{\mathrm{T}}(U)^{1 / 2} U^{4}\left(\frac{R_{\mathrm{D} 4}}{U}\right)^{3 / 2} \partial_{U} \varphi\right\}-2 k(U)^{1 / 2} f_{\mathrm{T}}(U)^{-1 / 2} U^{4}\left(\frac{R_{\mathrm{D} 4}}{U}\right)^{9 / 2} \partial_{0}^{2} \varphi \\
& +2 \kappa^{2} k(U)^{1 / 2} f_{\mathrm{T}}(U)^{1 / 2} U^{4}\left(\frac{R_{\mathrm{D} 4}}{U}\right)^{3 / 2} \frac{N(\Theta, U)^{2}}{\left(U / U_{0}\right)^{5}} \varphi=0 .
\end{aligned}
$$

In deriving the equation of motion, we have put $\varphi=\partial_{0} A_{1}$ and taken the Coulomb gauge $A_{0} \approx 0$. We expand the field $\varphi$ in terms of the complete set $\left\{\psi_{n}(U)\right\} \quad(n=1,2,3, \ldots)$ as $\varphi(t, U)=\sum_{n} v^{(n)}(t) \psi_{n}(U)$. The masses of $v^{(n)}$ are defined by

$$
\partial_{0}^{2} v^{(n)}+m_{n}^{2} v^{(n)}=0
$$


Using the equations (3.17), we derive the equation for the modes $\psi_{n}$

$$
\begin{aligned}
& u^{1 / 2} k(u)^{-1 / 2} f_{\mathrm{T}}(u)^{1 / 2} \partial_{u}\left\{u^{5 / 2} k(u)^{-1 / 2} f_{\mathrm{T}}(u)^{1 / 2} \partial_{u} \psi_{n}\right\} \\
& +\left[\frac{R_{\mathrm{D} 4}^{3}}{U_{0}} m_{n}^{2}+4 u^{-2} f_{\mathrm{T}}(u) N(\Theta, u)^{2}\right]=0
\end{aligned}
$$

where we have used the dimensionless variables $u=U / U_{0}$. The functions $f_{\mathrm{T}}(u), k(u)$ and $N(\Theta, u)$ are rewritten with respect to the dimensionless variables $u$ as follows;

$$
\begin{aligned}
& f_{\mathrm{T}}(u)=1-\left(\frac{u_{\mathrm{T}}}{u}\right)^{3}, \quad k(u)=\frac{u^{8}}{u^{8} f_{\mathrm{T}}(u)-f_{\mathrm{T}}(1)}, \\
& N(\Theta, u)=\frac{\Theta^{3 / 2} u^{3}}{1+\Theta^{3} u^{3}},
\end{aligned}
$$

where $u_{\mathrm{T}}=U_{\mathrm{T}} / U_{0}$ is a dimensionless parameter.

Eq. (3.19) is similar to the equation at finite temperature case [?]. The difference is the appearance of extra term $N(\Theta, u)$ in the mass term. It is convenient to decompose Eq. (3.19) into the first-order differential equations as

$$
\left\{\begin{array}{c}
\partial_{z} \psi_{n}(z)=\frac{2}{3} \frac{z\left(1+z^{2}\right)^{1 / 3}}{\left\{\left(1+z^{2}\right)^{8 / 3}-1-u_{\mathrm{T}}^{3}\left(\left(1+z^{2}\right)^{5 / 3}-1\right)\right\}^{1 / 2}\left(1+z^{2}-u_{\mathrm{T}}^{3}\right)^{1 / 2}} \zeta_{n}(z) \\
\partial_{z} \zeta_{n}(z)=-\frac{2}{3} \frac{z\left(1+z^{2}\right)}{\left\{\left(1+z^{2}\right)^{8 / 3}-1-u_{\mathrm{T}}^{3}\left(\left(1+z^{2}\right)^{5 / 3}-1\right)\right\}^{1 / 2}\left(1+z^{2}-u_{\mathrm{T}}^{3}\right)^{1 / 2}} \\
\quad \times\left[\lambda_{n}+\frac{4 \Theta^{3}\left(1+z^{2}-u_{\mathrm{T}}^{3}\right)\left(1+z^{2}\right)^{1 / 3}}{\left(1+\Theta^{3}\left(1+z^{2}\right)\right)^{2}}\right] \psi_{n}(z)
\end{array}\right.
$$

where $\lambda_{n}=\frac{R_{\mathrm{D} 4}^{3}}{U_{0}} m_{n}^{2}$ and $\zeta_{n}(z)=\frac{3}{2} \frac{\left\{\left(1+z^{2}\right)^{8 / 3}-1-u_{\mathrm{T}}^{3}\left(\left(1+z^{2}\right)^{5 / 3}-1\right)\right\}^{1 / 2}}{z\left(1+z^{2}\right)^{1 / 3}} \partial_{z} \psi_{n}(z)$. In deriving these equations, we have used the new variable $z^{2}=u^{3}-1$. As Eqs. (3.20) are symmetric for the transform $z \rightarrow-z, \psi_{n}(z)$ is even or odd function in $z$-variable. We can obtain the value of dimensionless parameter $\lambda_{n}$ from Eq. (3.20) under the regularity condition : $\psi_{n}(0)=0$ or $\partial_{z} \psi_{n}(0)=0$.

The asymptotic distance $L=\int d \tau$ between the position of $\mathrm{D} 8$ and anti-D8-branes is rewritten by using the expression (3.9) as

$$
L=\left(\frac{R_{\mathrm{D} 4}^{3}}{U_{0}}\right)^{1 / 2} K\left(u_{\mathrm{T}}\right), \quad K\left(u_{\mathrm{T}}\right) \equiv 2 \int_{1}^{\infty} d u \frac{u^{-3 / 2}}{\sqrt{f_{\mathrm{T}}(u)} \sqrt{\frac{f_{\mathrm{T}}(u)}{f_{\mathrm{T}}(1)} u^{8}-1}} .
$$

Recall that the temperature $T$ is expressed in terms of the parameters $u_{\mathrm{T}}$ and $R_{\mathrm{D} 4}$ :

$$
T=\frac{3}{4 \pi}\left(\frac{U_{\mathrm{T}}}{R_{\mathrm{D} 4}{ }^{3}}\right)^{1 / 2}=\frac{3}{4 \pi} \sqrt{u_{\mathrm{T}}}\left(\frac{U_{0}}{R_{\mathrm{D} 4}{ }^{3}}\right)^{1 / 2},
$$


where $U_{0}$ is the minimal point of the connected probe brane configuration, which can be related to the asymptotic distance $L$. From (3.21) and (3.22), we can rewrite the temperature $T$ in terms of the asymptotic distance $L$ :

$$
T\left(u_{\mathrm{T}}\right)=\frac{3}{4 \pi} \sqrt{u_{\mathrm{T}}} \frac{K\left(u_{\mathrm{T}}\right)}{L} .
$$

The ratio of $T$ to the confinement/deconfinement critical temperature $T_{c}=\frac{1}{2 \pi R}$ is

$$
\frac{T\left(u_{\mathrm{T}}\right)}{T_{c}}=\frac{3}{2} \sqrt{u_{\mathrm{T}}} K\left(u_{\mathrm{T}}\right) \frac{R}{L} .
$$

Substituting the $\chi \mathrm{SB} / \mathrm{SB}$ critical temperature $T\left(u_{\mathrm{T}}=0.73572\right)$ into (3.24), we have

$$
\frac{T\left(u_{\mathrm{T}}=0.73572\right)}{T_{\mathrm{c}}} \cong 0.97 \frac{R}{L}
$$

When the ratio (3.24) is larger than 1 , that is $L / R<0.97$, there is an intermediate-temperature phase [17]. When we put $L / R=0.4$, the intermediate-temperature phase occurs in the temperature range $1<T\left(u_{\mathrm{T}}\right) / T_{\mathrm{c}}<2.42$.

The mass squared of the mesons are expressed in terms of the eigenvalues $\lambda_{n}$ in (3.20) by using (3.21):

$$
m_{n}{ }^{2}=\frac{U_{0}}{R_{\mathrm{D} 4}{ }^{3}} \lambda_{n}=\frac{K\left(u_{\mathrm{T}}\right)^{2}}{L^{2}} \lambda_{n}
$$

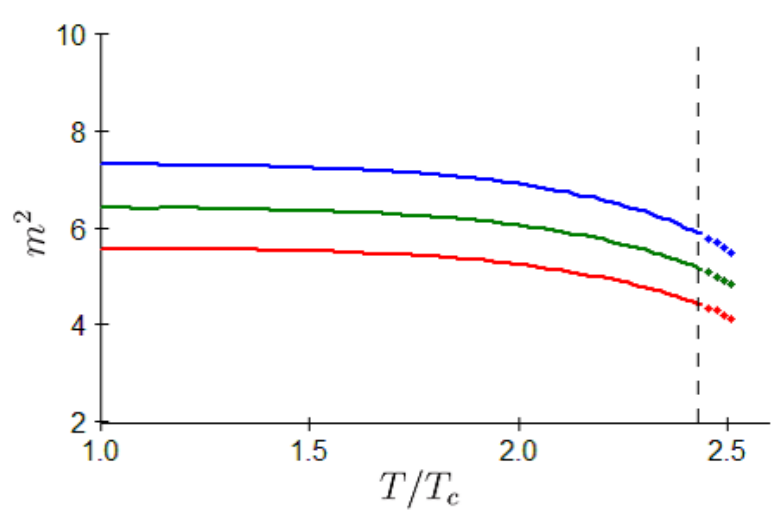

$\rho$-meson

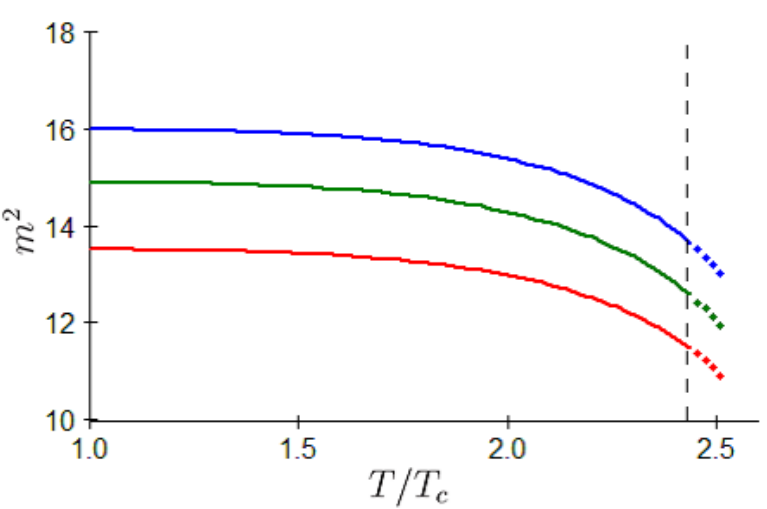

$a_{1}$-meson

Fig. 3 Masses squared of the lightest $\rho$ (left)- and $a_{1}$ (right)- mesons as a function of temperature $T$ in the intermediate-temperature regime. From the top(blue) to the bottom(red) line in the left hand side graph represents the value of $\Theta$ for $0.0,1.5,0.1$, respectively. From the top(blue) to the bottom(red) line in the right hand side graph represents the value of $\Theta$ for 0.0 , $1.2,0.1$, respectively. 
The mass squared of the mesons depends on the temperature $T$. The temperature dependence of mass squared for the lightest $\rho$ (vector)- and $a_{1}$ (pseudovector)- mesons in the intermediate-temperature regime are shown in figure 3.

We can observe that the masses of light vector- and pseudovector-meson decrease with increasing temperature $T$. This is consistent with the result of the holographic model for mesons [27, 24] and lattice calculation [28]. In addition, the masses of meson reduce over the whole area in the intermediate-temperature regime by the space noncommutativity. In this sense, the noncommutativity parameter $\theta$ plays the role like the temperature $T$. However, the dependence for the noncommutativity parameter of the masses is not monotonic. The noncommutativity parameter dependence of the lightest $\rho$ (vector)- and $a_{1}$ (pseudovector)- mesons in the intermediate- temperature regime are shown in figure 4.

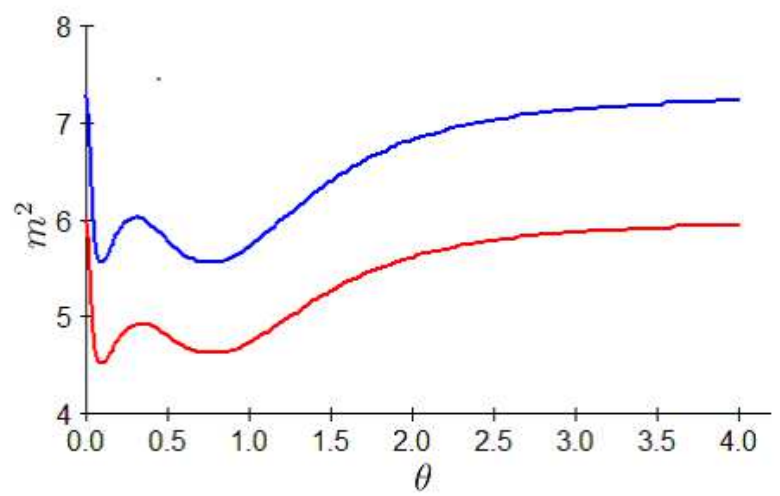

$\rho$-meson

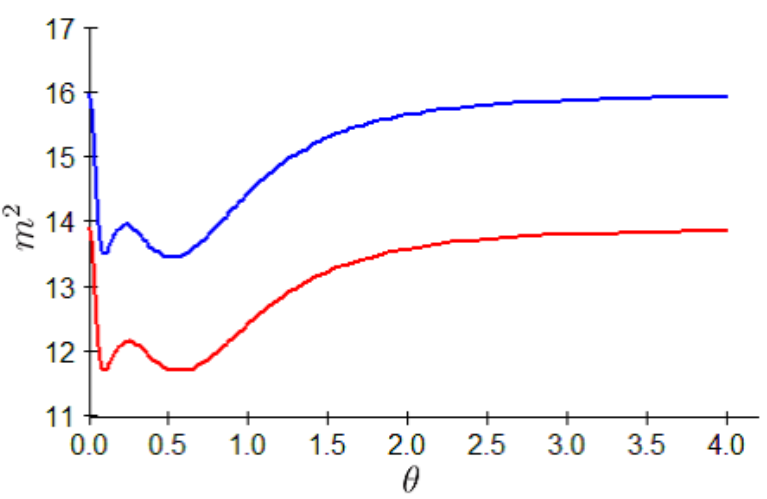

$a_{1}$-meson

Fig. 4 Masses squared of the lightest $\rho$ (left)- and $a_{1}$ (right)- mesons as a function of noncommutativity parameter $\Theta$ in the intermediate-temperature regime. The lower(red) and upper(blue) line in the each side graph represents the value of $T$ for 1.2, 2.4, respectively.

There is a little difference of the noncommutativity parameter dependence between the masses of $\rho-$ and $a_{1}$ mesons. However, there is a slight difference of the noncommutativity parameter dependence between the masses of high and low temperature. We notice that the masses of light vector- and pseudovector-meson return to the commutative case as the noncommutativity parameter becomes larger. This feature is common with the gravity dual of noncommutative gauge theories [14, 13, 29]. The mass trajectories for the ground and excited state in the intermediate-temperature regime are shown in figure 5. 

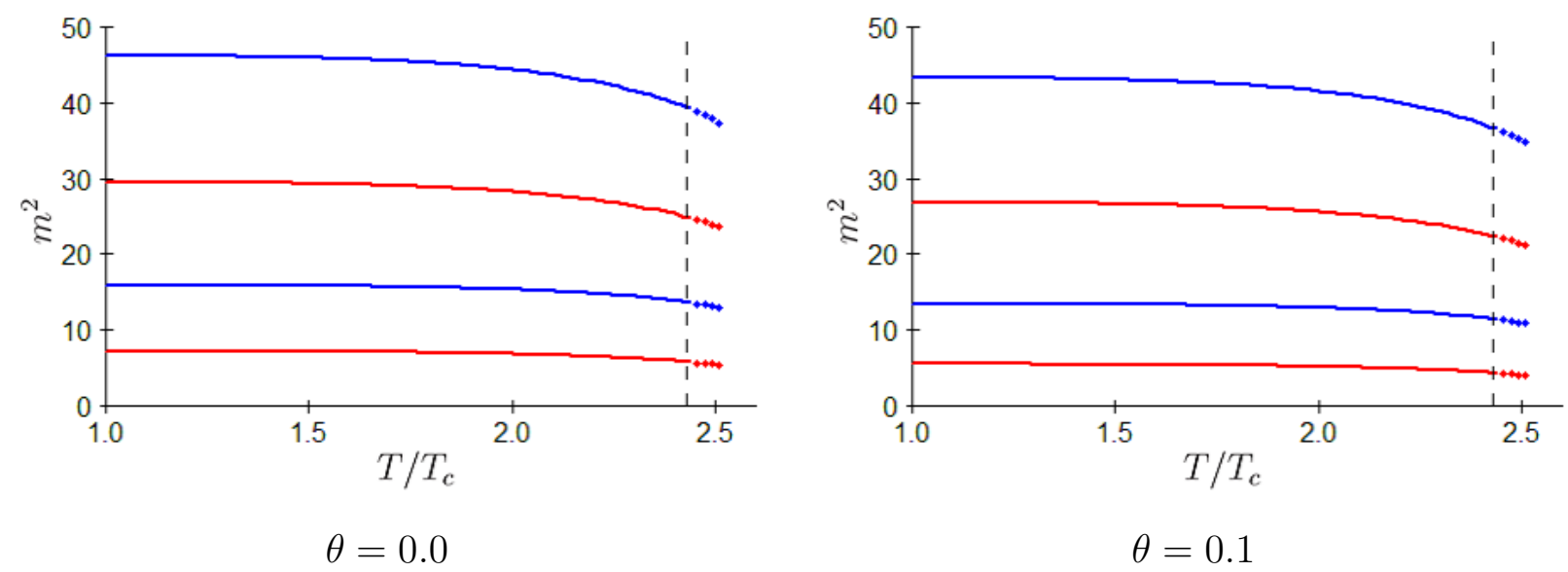

Fig. 5 Masses squared of the ground and first excited state of mesons as a function of temperature $T$ in the intermediate-temperature regime. The commutative $(\theta=0.0)$ and noncommutative $(\theta=0.0)$ case is illustrated on the left and right hand side of the figure, respectively. From the bottom to the top line in the both sides graph represents the ground state of $\rho$-meson, the ground state of $a_{1}$-meson, the first excited state of $\rho$-meson, the first excited state of $a_{1}$-meson, respectively.

In noncommutative case, the masses of meson decrease as temperature increase over the whole area in the intermediate-temperature regime not only the ground state but also the excited states. However, the mass differences of the excited state of meson between the commutative case and the noncommutative case in the same temperature are larger than that of the ground state. The mass trajectories for the ground and excited state in the noncommutative case become flat than that in the commutative case.

\section{Conclusions}

In this paper, we have constructed a noncommutative deformation of the holographic QCD (Sakai-Sugimoto) model after the prescription of Alishahiha et al [9] and Arean et al. [14] and evaluated the mass spectrum of low spin vector mesons at finite temperature. The masses of light vector- and pseudovector-meson in the noncommutative holographic QCD model reduces over the whole area in the intermediate-temperature regime compared to the commutative case. However, the space noncommutativity does not change the properties of the temperature dependence for the mass spectrum of low spin mesons [27, 24]. The masses of meson also decrease with increasing temperature in noncommutative case. The variation of masses of meson with temperature becomes small in noncommutative case. The intermediate-temperature phase is easy to be realized in the noncommutative deformation of the holographic QCD model [29]. The smallness of mass variation with temperature, namely, the hardness of melting meson may 
be related with the property of phase in the noncommutative deformation of the holographic QCD model.

The magnitude of noncommutativity parameter $\theta$ denotes the degree of space noncommutativity in the noncommutative theory. The noncommutative deformation of the holographic QCD model reduces to the commutative one when the noncommutativity parameter tends to zero. However, the mass spectrum of low spin mesons in the noncommutative theory result in that of the commutative theory under the large theta limit. This fact shows that the noncommutative deformation of the holographic QCD model also reduces to the commutative model when the noncommutativity parameter approaches infinity. This property is common to other noncommutative deformation of holographic models. In addition, it is also common with other noncommutative deformation of holographic models that noncommutativity is introduced via the WZ terms in the effective action for probe brane [14, 29].

It has been shown that the dissociation temperature of mesons with large spin is spin dependent and higher spin mesons have a tendency to melt at lower temperature in the context of the holographic QCD model [24, 25]. To investigate how the noncommutativity have an effect on the properties of meson melting is of particular interest. We can confirm that the dissociation temperature of large spin mesons also depend on the noncommutativity parameter [33.

The UV/IR mixing is well known as distinctive features of noncommutative field theories $\underline{6}$, 31, 32]. The UV/IR mixing appears to be the qualitative difference between ordinary and noncommutative field theory. The difference in properties of meson mass spectrum between ordinary and noncommutative deformation of QCD might be related to the UV/IR mixing. We hope to discuss this subject in the future.

\section{Acknowledgments}

Y.O. was supported by MEXT grant to aid women researchers. T.N. would like to thank members of the Physics Department at College of Engineering, Nihon University for their encouragements. The authors are grateful to A. Sugamoto for useful discussions and comments. 


\section{References}

[1] A. Connes, M. R. Douglas, A. Schwarz, Noncommutative Geometry and Matrix Theory: Compactification on Tori, JHEP 02 (1998) 003, arXiv:hep-th/9711162.

[2] M. R. Douglas and C. Hull, D-branes and the Noncommutative Torus, JHEP 02 (1998) 008, arXiv:hep-th/9711165.

[3] F. Ardalan, H. Arfaei and M.M. Sheikh-Jabbari, Noncommutative Geometry From Strings and Branes, JHEP 02 (1999) 016, arXiv:hep-th/9810072.

[4] M.M. Sheikh-Jabbari, Super Yang-Mills Theory on Noncommutative Torus from Open Strings Interactions, Phys. Lett. B450 (1999) 119-125, arXiv:hep-th/9810179.

[5] N. Seiberg and E. Witten, String Theory and Noncommutative Geometry, JHEP 9909 (1999) 032, arXiv:hep-th/9908142.

[6] S. Minwalla, M. V. Raamsdonk and N. Seiberg, Noncommutative perturbative dynamics JHEP 0002 (2000) 020, arXiv:hep-th/9912072

[7] A. Hashimoto and N. Itzhaki, Non-Commutative Yang-Mills and the AdS/CFT Correspondence, Phys. Lett. B465 (1999) 142-147, arXiv:hep-th/9907166.

[8] J. M. Maldacena and J. G. Russo, Large N Limit of Non-Commutative Gauge Theories, JHEP 9909 (1999) 025, arXiv:hep-th/9908134.

[9] M. Alishahiha, Y. Oz and M.M. Sheikh-Jabbari, Supergravity and Large N Noncommutative Field Theories, JHEP 9911 (1999) 007, arXiv:hep-th/9909215.

[10] A. Dhar and Y. Kitazawa, "Wilson loops in strongly coupled noncommutative gauge theories," Phys. Rev. D63 (2001) 125005, arXiv:hep-th/0010256.

[11] S. Lee and S. Sin, "Wilson Loop and Dimensional Reduction in Non-Commutative Gauge Theories," Phys. Rev. D64 (2001) 086002, arXiv:hep-th/0104232.

[12] H. Takahashi, T. Nakajima and K. Suzuki, "D1/D5 system and Wilson Loops in (Non-)commutative Gauge Theories," Phys. Lett. B546 (2002) 273, arXiv:hep-th/0206081.

[13] T. Nakajima, K. Suzuki and H. Takahashi, "Glueball mass spectra for supergravity duals of noncommutative gauge theories," JHEP 0601 (2006) 016, arXiv:hep-th/0508054. 
[14] D. Arean, A. Paredes and A.V. Ramallo, Adding flavor to the gravity dual of noncommutative gauge theories, JHEP 0508 (2005) 017, arXiv:hep-th/0505181.

[15] T. Sakai and S. Sugimoto, Low energy hadron physics in holographic QCD, Prog. Theor. Phys. 113 (2005) 843-882, arXiv:hep-th/0412141.

[16] T. Sakai and S. Sugimoto, More on a holographic dual of QCD, Prog. Theor. Phys. 114 (2006) 1083-1118, arXiv:hep-th/0507073.

[17] O. Aharony, J. Sonnenschein and S. Yankielowicz, A holographic model of deconfinement and chiral symmetry restoration, Annals Phys. 322 (2007) 1420-1443, arXiv:hep-th/0604161.

[18] A. Parnachev and D. A. Sahakyan, Chiral Phase Transition from String Theory, Phys.Rev.Lett. 97 (2006) 111601, arXiv:hep-th/0604173,

[19] N. Horigome and Y. Tanii, Holographic chiral phase transition with chemical potential, JHEP 0701 (2007) 072, arXiv:hep-th/0608198.

[20] A. Parnachev and D. A. Sahakyan, Photoemission with Chemical Potential from QCD Gravity Dual, Nucl. Phys. B768 (2007) 177-192, arXiv:hep-th/0610247.

[21] D. Yamada, Sakai-Sugimoto Model at High Density, JHEP 0810 (2008) 020, arXiv:0707.0101[hep-th].

[22] O. Bergman, G. Lifschytz and M. Lippert, Holographic Nuclear Physics, JHEP 0711 (2007) 056, arXiv:0708.0326[hep-th].

[23] M. Rozali, H.-H. Shieh, M. V. Raamsdonk and J. Wu, Cold Nuclear Matter In Holographic QCD, JHEP 0801 (2008) 053, arXiv:0708.1322[hep-th].

[24] K. Peeters, J. Sonnenschein and M. Zamaklar, Holographic melting and related properties of mesons in a quark gluon plasma, Phys. Rev. D74 (2006) 106008, arXiv:hep-th/0606195.

[25] K. Peeters, J. Sonnenschein and M. Zamaklar, Holographic decays of large-spin mesons, JHEP 0602 (2006) 009, arXiv:hep-th/0511044.

[26] K. Y. Kim, S.-j. Sin and I. Zahed, Dense Hadronic Matter in Holographic QCD, arXiv:hep-th/0608046.

[27] K. Ghoroku and M. Yahiro, Holographic Model for Mesons at Finite Temperature, Phys.Rev. D73 (2006) 125010, arXiv:hep-ph/0512289. 
[28] F. Karsch, Lattice QCD at Finite Temperature and Density, Nucl. Phys. Proc. Suppl. D83 (2000) 14 arXiv:hep-lat/9909006.

[29] T. Nakajima, Y. Ohtake and Kenji Suzuki, Chiral Symmetry Restoration in Holographic Noncommutative QCD, JHEP. 1109 (2011) 054, arXiv:1011.2906 [hep-th].

[30] M. Ali-Akbari, Non-commutative Holographic QCD and Jet Quenching Parameter, arXiv:1104.4924 [hep-th].

[31] M. R. Douglas and N. A. Nekrasov, Noncommutative Field Theory, Rev. Mod. Phys. 73 (2001) 977-1029, arXiv:hep-th/0106048.

[32] R.J. Szabo, Quantum field theory on noncommutative spaces, Phys. Rept. 378 (2003) 207-299, arXiv:hep-th/0109162.

[33] T. Nakajima, Y. Ohtake and Kenji Suzuki, in preparation. 